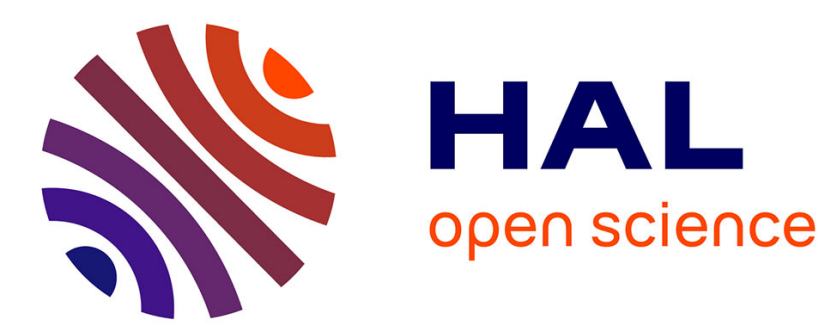

\title{
Methodology for automatic recovering of 3D partitions from unstitched faces of non-manifold CAD models
}

Alexei Mikchevitch, Jean-Philippe Pernot

\section{To cite this version:}

Alexei Mikchevitch, Jean-Philippe Pernot. Methodology for automatic recovering of 3D partitions from unstitched faces of non-manifold CAD models. Engineering with computers, 2013, pp.1-12. 10.1007/s00366-013-0325-y . hal-01086087

\section{HAL Id: hal-01086087 https://hal.science/hal-01086087}

Submitted on 13 Feb 2015

HAL is a multi-disciplinary open access archive for the deposit and dissemination of scientific research documents, whether they are published or not. The documents may come from teaching and research institutions in France or abroad, or from public or private research centers.
L'archive ouverte pluridisciplinaire HAL, est destinée au dépôt et à la diffusion de documents scientifiques de niveau recherche, publiés ou non, émanant des établissements d'enseignement et de recherche français ou étrangers, des laboratoires publics ou privés. 


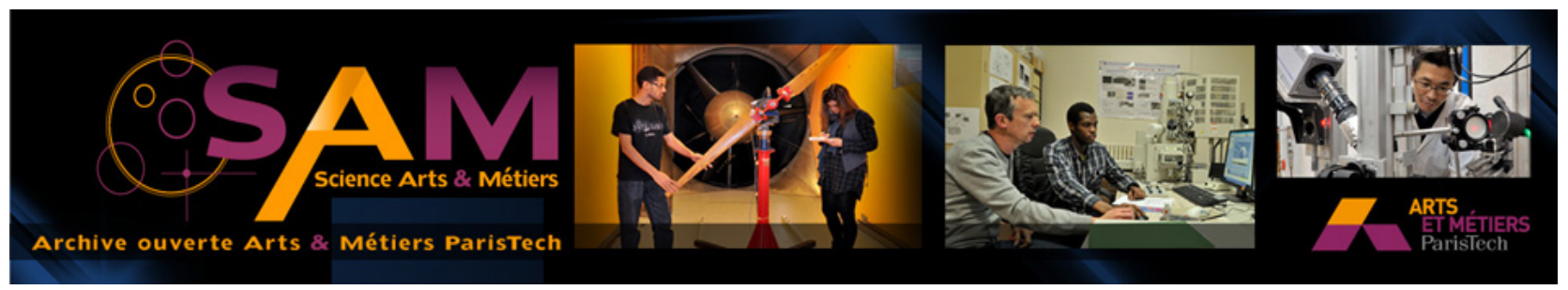

Science Arts \& Métiers (SAM)

is an open access repository that collects the work of Arts et Métiers ParisTech researchers and makes it freely available over the web where possible.

This is an author-deposited version published in: http://sam.ensam.eu Handle ID: .http://hdl.handle.net/10985/8965

\section{To cite this version :}

Alexei MIKCHEVITCH, Jean-Philippe PERNOT - Methodology for automatic recovering of 3D partitions from unstitched faces of non-manifold CAD models - Engineering with computers p.1-12 $-2013$ 


\title{
Methodology for automatic recovering of 3D partitions from unstitched faces of non-manifold CAD models
}

\author{
Alexei Mikchevitch · Jean-Philippe Pernot
}

\begin{abstract}
Data exchanges between different software are currently used in industry to speed up the preparation of digital prototypes for finite element analysis (FEA). Unfortunately, due to data loss, the yield of the transfer of manifold models rarely reaches 1 . In the case of nonmanifold models, the transfer results are even less satisfactory. This is particularly true for partitioned 3D models: during the data transfer based on the well-known exchange formats, all 3D partitions are generally lost. Partitions are mainly used for preparing mesh models required for advanced FEA: mapped meshing, material separation, definition of specific boundary conditions, etc. This paper sets up a methodology to automatically recover 3D partitions from exported non-manifold CAD models to increase the yield of the data exchange. Our fully automatic approach is based on three steps. First, starting from a set of potentially disconnected faces, the CAD model is stitched. Then, the shells used to create the $3 \mathrm{D}$ partitions are recovered using an iterative propagation strategy which starts from the so-called manifold vertices. Finally, using the identified closed shells, the 3D partitions can be reconstructed. The proposed methodology has been validated on academic as well as industrial examples.
\end{abstract}

\footnotetext{
A. Mikchevitch

Research and Development Direction, EDF Group, 1 Avenue du Général de Gaulle, 92141 Clamart, France

e-mail: alexei.mikchevitch@edf.fr

J.-P. Pernot $(\square)$

Arts et Métiers ParisTech, Systems and Information Sciences Laboratory, UMR CNRS 7296, 2 cours des Arts et Métiers, 13617 Aix-en-Provence, France

e-mail: jean-philippe.pernot@ensam.eu
}

Keywords CAD modelling - Data exchange $\cdot$ Nonmanifold models $\cdot 3 \mathrm{D}$ partition

\section{Introduction}

Nowadays, products are classically designed and/or improved following several optimisation loops that successively create/modify CAD models, create/update finite element (FE) meshes, and simulate products' behaviour according to the specific boundary conditions and behaviour laws. During this iterative process, multiple tools are often used in industrial practice. For example, a CAD model can be designed and modified using a software A and meshed using the advanced meshing capabilities of a software B to finally end in a third software C for the FE simulation. When the simulation results are not satisfactory, the CAD models are modified, the meshes are updated and the simulations rerun. Despite a general tendency to set up new product modelling framework for lifecycle management, the interoperability between software is still mainly ensured by numerous data exchanges based on neutral formats [1]. However, due to data loss of different nature [2], the yield of these exchanges is often low, especially when considering exchanges of complex nonmanifold CAD models.

As defined in [3], for regular solids, a small enough sphere around every point on the boundary is divided into two parts, one inside and one outside, and thus defining the interior and exterior of the 3D object. Non-manifold models do not follow this rule. Figure 1 presents such an example: a holed half-cylinder decomposed in four 3D partitions using non-manifold faces. A non-manifold face is bounded by at least one non-manifold edge, i.e. an edge connected to more than two faces (three in the present 
Fig. 1 Example of nonmanifold CAD model. a Solid decomposed into four 3D partitions. b Internal nonmanifold faces bounding 3D partitions of the solid

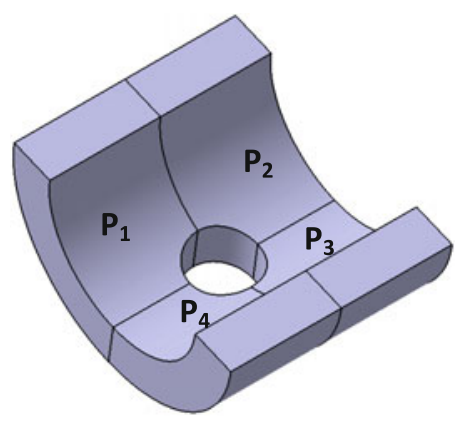

(a)

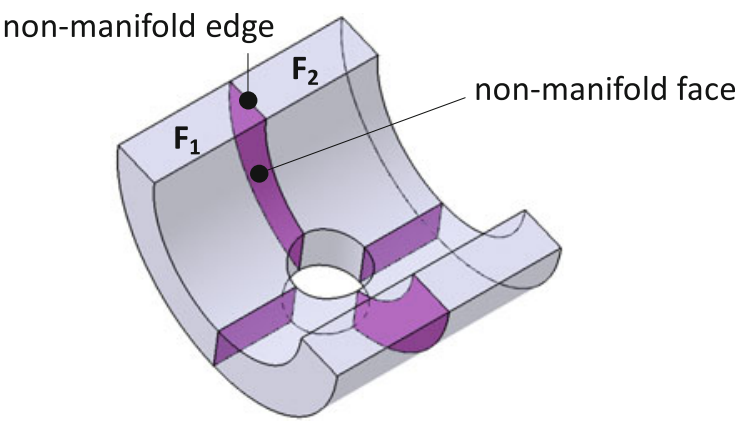

(b) case). In other words, a non-manifold face does not split the 3D space with an inside and an outside, or one can also define it as a face used to split the model in two adjacent partitions.

Today, the exchanges of non-manifold models between CAD environments are not fully supported by the existing neutral formats. Thus, 3D partitions are often lost and their recovery requires multiple manipulations of low-level geometric entities: re-creation of closed shells, re-definition of solids and partition tools, reconstruction of partitions properly saying. Actually, most of the time, the geometric models are exploded during the data exchange and their faces have to be stitched before starting the identification of the shells bounding the various $3 \mathrm{D}$ partitions to be reconstructed. These extra modifications may rapidly become tedious and time-consuming when considering geometric models made of several hundreds of partitions. They require a deep knowledge of the underlying mathematical concepts and properties. Sometime, it is even faster to reconstruct the geometric models starting from scratch than to try to import them. However, it is not acceptable to stop the integrated design workflow with interactive tasks preventing information propagation.

The reducing of CAD modelling time is particularly relevant for fast studies applied to maintenance/life cycle problems where it is critical to provide quickly the optimised solution and to ensure its effectiveness $[4,5]$. In the context of industrial maintenance, the product is already designed, and companies currently practice the re-use of existing non-manifold CAD models via exchange data formats to accelerate the study/improvement of the product during its exploitation. Therefore, the efficient import of non-manifold models enriched by the so-called 3D partitions used for fast finite element analysis (FEA) of the product's behaviour is an important aspect from industrial application point of view.

In this paper, a methodology for automatic recovering 3D partitions from unstitched faces of non-manifold CAD models is proposed. The algorithms have been implemented and validated within the SALOME ${ }^{\circledR}$ platform [6]. The paper is organised as follows. First, the industrial needs and methods for modelling non-manifold objects are discussed. In particular, we focus on non-manifold models enriched by the so-called 3D partitions. Other non-manifold configurations are not covered. Section 3 depicts the works related to exchange of manifold CAD models as well as non-manifold ones currently required for advanced FEA. Section 4 introduces the overall methodology of for automatic reconstructing 3D partitions and gives some details of our algorithm. Section 5 illustrates the proposed methodology on academic and industrial examples, and Sect. 6 concludes the paper.

\section{On the use of non-manifold CAD models}

\subsection{Modelling of non-manifold objects}

There are many industrial examples in design and maintenance analysis where, to simulate complex physical phenomena or objects (e.g. damaged part behaviour, contact problem, interaction of the structure with its environment, etc.), engineers have to proceed with a modelling strategy involving particular operators [7-9]. These examples show that engineers need operators to build a socalled non-manifold geometry. To simulate the behaviour of a multi-material structure or to prepare the model for mapped meshing, the so-called partition operators are needed to divide the model into several connected areas. From industrial experience, Rossignac and Requicha [10] propose to extend the conventional solid modelling method known as constructive solid geometry (CSG) by introducing a concept of constructive non-regularised geometry to support more general representation of geometric objects.

Various approaches have emerged to model non-manifold objects. Boundary-based data structures for such objects have been proposed in [11-13]. In most of these works, the modelling of non-manifold shapes is based on a decomposition of space into cells of various dimensions (e.g. volumes, faces, edges). Weiler [14] proposes a modelling of non-manifold 3D objects using a radial-edge data structure allowing describing face-edge and edge-vertex 
incidence relations. De Floriani and Hui [15] propose an indexed data structure with adjacencies useful for nonmanifold modelling based on 3D simplicial complexes. Another method to represent non-manifold shape consists in its decomposition into manifold or regular sub-shapes. Desaulnier and Stewart [16] propose a representation scheme based on a decomposition of solid object into regular parts (r-sets) that provides topological information about the object. The so-called selective geometric complexes (SGCs) introduced in [17] can describe non-manifold 3D objects through cell complexes. This paper provides a framework for representing non-manifold objects having internal structures and incomplete boundaries as isolated points, edges, faces, and solids with internal structures and cracks. SGCs are composed of collections of mutually disjoints cells, which are open connected subsets of $n$-dimensional manifolds and generalise the concept of edges, faces, and vertices used in solid modellers. In SGCs, cells and their mutual adjacencies are encoded in an incidence graph. Hui et al. [18] propose to decompose the non-manifold object into regular parts by splitting the shape at those elements (vertices, edges, faces) where singularities occur. For 3D, decomposition into manifold parts may need to introduce artificial "cuts" through the object.

In addition, the apparition of different meshing methods has accompanied the emergence of CAD tools handling nonmanifold models. We can mention a meshing method by Marcheix and Gueorguieva [19] allowing creating triangular elements for non-manifold solid boundaries. Saxena et al. [20] propose an octree-based algorithm to mesh a non-manifold domain with mixed tetrahedral and triangular elements. Their paper focuses on mesh generation issues in the context of FE simulation of multi-material objects. Zhang et al. [21] describe an automatic approach to mesh a composite domain made up of heterogeneous materials. The boundaries of these material regions form non-manifold surfaces.

Recently, a method for meshing 3D CAD assemblies has been proposed [22]. This method allows generating unstructured hexahedral meshes with preservation of boundaries shared by two parts being in contact. The boundaries are recovered using a node relocation technique applied throughout the contact surface detected. The mesh smoothing is combined with a local optimisation to improve the mesh quality. The boundary reconstruction strategy based on a mesh processing increases significantly the memory and CPU time, particularly in the case of large models.

\subsection{Use of partitioned non-manifold models}

Partitioning a CAD model corresponds to one class of nonmanifold geometric operations and consists in a sub-division of the model into different regions using a so-called partition tool (e.g. face, edge) to prepare advanced mesh for complex FE modelling of the product's behaviour. In case of 2D topology, the result of such an operation is a set of connected surfaces having common boundaries that are edges. In case of 3D, initially regular solid is transformed after partitioning into a non-manifold 3D object representing a set of sub-volumes having common boundaries that are internal faces. From industrial practice, the partitions can be used to:

- Create mapped meshes required for FE simulation of cracks or thin structures modelled as solids (e.g. tubelike structures), etc. The mapped mesh allows avoiding the singular stress problem on the crack tip, controlling the number of elements through the thickness of thin structures, etc.,

- Create a priori adaptative-free meshes. For example, the model can be sub-divided into different areas where the mesh refinement may be defined relatively to the zone of interest: small-size elements are created in problematic zone for more accurate analysis, and progressive mesh is generated around this zone to reduce the global size of the discretised model,

- Create groups of FE entities (e.g. sets of faces, edges, nodes) to support the semantics of the FEA (e.g. boundary conditions and/or loads) assigned to a given area. The notion of mesh groups is currently used in industrial CAD and FE simulation tools for preparing advanced FE models,

- Differentiate parts of a model sharing various mechanical characteristics, e.g. heterogeneous behaviour laws, as well as multiple geometric characteristics, e.g. double entities along a crack or surface of interaction between a solid and a fluid, etc. Physical characteristics are defined using 3D mesh entity groups (3D submeshes) delimited by faces of corresponding partitions.

Actually, a given CAD model may also contain multiple partitions dedicated to different and completely distinct FE simulations that is very practical from industrial point of view, namely, for parametric studies. Thus, hundreds of partitions may be combined in a unique CAD model. This point is illustrated on the academic example of Fig. 2 wherein the CAD model of a quarter-tube is decomposed into three 3D partitions (Fig. 2a) that can be combined to prepare two different FEA (Fig. 2b). FE simulation cases can be distinguished by affecting (or not) the mechanical properties to given sub-solids corresponding to the $3 \mathrm{D}$ partitions $\mathbf{P}_{\mathbf{1}}$ and $\mathbf{P}_{\mathbf{2}}$.

In the present paper, solely the configurations involving 3D partitions defined by at least one non-manifold face have been considered. These configurations are problematic in terms of CAD data exchange: after import of partitioned 3D models, besides the classical data losses (e.g. 


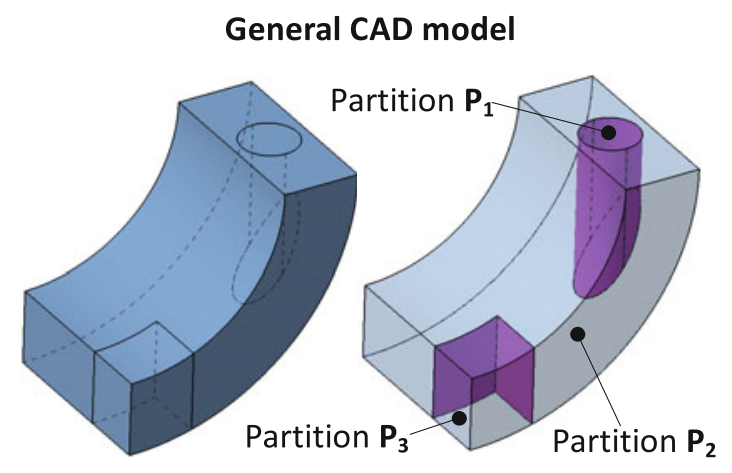

(a)
Simulation CAD models for FEA
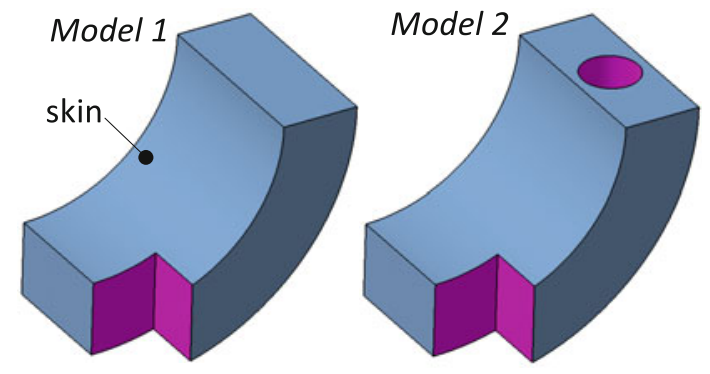

(b)

Fig. 2 Use of 3D partitions for parametric studies. a General CAD model of a quarter-tube decomposed into three 3D partitions. b Example of two distinct FE simulation models created from the general partitioned CAD model

history, constraints, etc.), the topology of the partitioned solid model is constantly lost, even if the chosen modelling tolerance is sufficient. Currently, the CAD systems manipulating non-manifold solids cannot automatically rebuild the volume of the imported partitioned models: at best, only some open shells are recovered. 3D partitions completely immersed within the solid, and therefore defined by manifold faces, have not been considered yet. However, it can be mentioned that the latter may be reduced to the configuration studied in this paper by adding supplementary partition faces within the 3D object.

\section{Exchanging and repairing CAD models: related works}

The aspects related to the interoperability between CAD systems as well as the methods for exchange, control and repair of models are widely studied in literature. Most problems in data exchange are due to both the differences between internal mathematical representation schemes and to the internal accuracies of the modelling kernels integrated in the CAD systems [2, 23, 24]. Data exchange problems can also arise from an inadequate geometric modelling of the original model. They can also be due to inappropriate choices of exchange formats or specific transfer options which may strongly depend on the CAD systems. These problems may be difficult to identify.

In CAD data exchange, the loss of information about the construction of geometric object (history, parameters, and constraints) and its topology is recurrent. In particular, imported 3D model may not be reconstructed in the receiving system if gaps or overlaps occur due to inaccuracy during the solid translation. The shape of the object may be transferred but the topological information required for considering the object as a regular solid is no longer valid. Topology errors take place when topological entities are wrongly defined (i.e. in presence of non-manifold edge or interior face) or inaccurately connected (i.e. shell is not closed if gaps exceeding the model tolerance occur). Many previous researches have addressed both the diagnostic and correction of geometric and topological problems. Krause et al. [24] analyse translation problems with IGES format, gives examples of topology errors and proposes a data processing system able to detect the face adjacency, to calculate the topological relations between faces, and to repair gaps and overlaps. Ficco et al. [25] develop a semiautomatic method for identifying and correcting errors while re-constructing solids from surface-based models. In [26], some aspects related to IGES and STEP formats are pointed out. The authors highlight that the problems originate from implementation of the format interface and that the format specification is open to individual interpretation. Sangole et al. [27] propose a manual repair scheme for STEP files and describe the export/import accuracies.

Due to stable mathematical behaviour, realisation efficiency and low hardware requirements for processing of large CAD models, NURBS Boundary Representation (BREP) models can be suitable for modelling of complex structures [28]. However, such models can contain various errors (e.g. gaps, incorrect topology of trimming curves, etc.) caused by the lack of constraints on the global continuity of the object's boundary [29]. These errors can be introduced by the CAD engine itself and/or via data exchanges. Mezentsev et al. [29] give an overview of a NURBS-based CAD data repair methods and algorithms. For effective surface meshing, the authors propose to extend the concept of CAD repair, classically based on the correction of geometric and topological errors, by detection of the so-called badly meshable geometries corresponding to geometrically valid elements (as taper or small faces, long thin faces, warped faces, etc.) generating FE analysis problems.

Mesh-based approaches can also be applied to CAD repair problem: they allow identifying geometric and topological errors on the surface by checking polygon 
connection [30]. Once found, the errors are fixed by performing various geometric operations on the surface: adding or removing vertices, modifying their locations, modifying the polygon connectivity. More information about mesh-based repairing methods can be found in [3034].

Current CAD systems generally incorporate functions to check and correct the imported models but, unfortunately, they do not automatically avoid information loss, especially when the models are complex. Gerbino [2] analyses how to repair the exported CAD model as well as how to prepare the model to prevent failure in data exchange via neutral formats. The author suggests transferring data only needed for a given application, for example, engineers can simplify the CAD model used for FEA, and to define the appropriate accuracy. The appropriate choice of geometric entities required for specific physical simulation is only possible if the FEA expert participates directly to the CAD modelling or model-preparing phases. Furthermore, what do we do if a given FEA necessitates a non-manifold modelling (e.g. internal surfaces or crack introduced into a solid), case for which current neutral formats are not expected? Automatic repairing of 3D model boundaries is difficult. Gerbino [2] proposes to remove the problematic surfaces and to recreate them into the receiving system. These extra operations require a deep knowledge of the modelling concepts. They may modify the design geometry and rapidly become time-consuming when models contain many non-manifold configurations.

Yang and Han [35] propose an approach to repairing CAD model errors that is based on a design history schema that can be extracted from the CAD model. The authors suggest using this design history schema to analyse the interdependency and parametric data of feature commands reconstructed through rule-based reasoning of an expert system. The CAD model correction system developed in [35] repairs the most current geometric errors (e.g. small faces, narrow regions), but does not address non-manifold configurations like partitioned 3D models containing internal faces.

Deduced from experience, Gu et al. [23] classify typical interoperability errors and provide a few guidelines for improving interoperability at the initial design stage. The question that arises here is how to handle 3D models of real structures already designed, for example, if we want to assess the lifecycle of the machinery or to improve its behaviour during the exploitation? In this case, it seems difficult to re-use the digital prototype of the existing machinery corresponding to the first design stages. Indeed, maintenance assessment studies are usually based on the real design $\mathrm{CAD}$ model adapted to a given study, e.g. by adding new features as cracks (if the machinery is damaged), 2D/3D partitions (to model the interaction of the equipment with its environment), etc. Such modifications may generate different geometric problems so that the obtained non-manifold model, transferred into simulation environment for meshing and FEA, may be difficultly exploitable.

It can also be mentioned that certain CAD systems do not enable the use of non-manifold models within their geometric kernel. Hence, the reconstruction of transferred non-manifold 3D models containing internal faces consists generally in removing or ignoring all internal faces (or other entities considered as anomalies from the geometric modelling point of view) and stitching of boundary faces to recreate the manifold solid. In most of the proposed approaches, non-manifold geometries are often considered as anomalies, which have to be corrected. Therefore, a specific treatment is necessary to rebuild imported nonmanifold $\mathrm{CAD}$ models required for advanced $\mathrm{FE}$ simulations.

Unlike manifold models, non-manifold ones do not divide the space into inside and outside, creating new challenges for non-manifold solid transferring and repairing [30]. In this paper, we do not pretend to work neither on the IGES nor the STEP norms. Instead, we propose a methodology to reconstruct automatically 3D partitions (equivalent to reconstruct the non-manifold 3D model) from an exploded set of faces recovered from a transferred non-manifold model (see Fig. 3). The problem of rebuilding such particular models cannot be handled using classical geometric modelling methods. Indeed, many CAD systems reconstruct the solid from boundary faces of the
Fig. 3 Explosion in faces when transferring a non-manifold model from a CAD system $A$ to another one $\mathrm{B}$

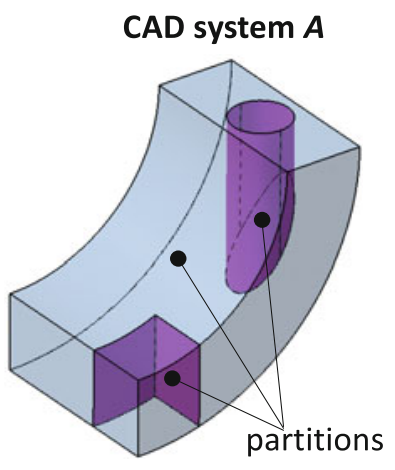

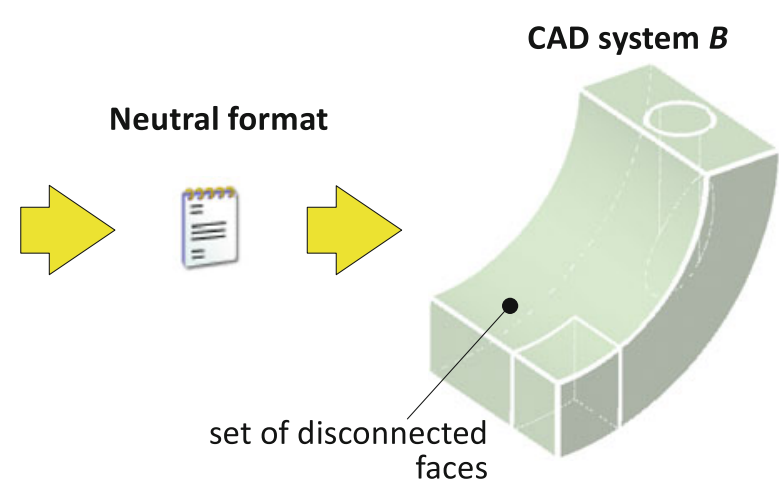


imported model and do not take into account the presence of internal faces required for partitioning the 3D model. First introduced in [36], our method is extended in the following section to circumvent this problem.

\section{Reconstructing 3D partitions}

\subsection{Overall methodology}

Starting from industrial practices identified through EDF's engineering projects, we focus on the exchange problem of non-manifold complex 3D models currently used for FE modelling when improving the mechanical behaviour of power production machinery. Actually, engineers use various systems to design, modify, mesh and simulate industrial structures, and they are asked to perform data transfers by taking advantage of advanced functionalities of given software in terms of geometric modelling, meshing or FE simulation. Non-manifold faces are usually introduced into solids to model complex objects (multi-material parts) or phenomena (cracks, contacts, mixed-physics interactions) as well as to prepare the CAD model for a specific meshing required for FE simulation and advanced post-processing of results. Currently, the geometric modelling of such nonmanifold configurations consists in introducing internal faces into regular solids that corresponds to a non-conventional partitioning operation. Our work aims to handle the exchange problems of non-manifold models enriched by $3 \mathrm{D}$ partitions to increase the transfer yield during the exchange of industrial CAD data between platforms able to manipulate non-conventional geometries (for example, I-DEAS $^{\circledR}$ and SALOME $^{\circledR}$ CAD systems). In this paper, other complex non-manifold configurations have not been considered (e.g. edges connected to faces by their end points) even if they are widely used to prepare CAD models to specific FE simulations (e.g. mixing of 1D, 2D and 3D mesh elements). In addition, it is here supposed to have edges of the CAD model connected to at least two edges, otherwise the algorithm generates non-closed shells to which a solid cannot be associated.

In the present paper, we propose an approach enabling the automatic reconstruction of 3D partitions of transferred CAD models starting from a set of disconnected faces imported from a file (such as IGES, STEP). To make the method more general, we suppose that the imported model has lost information about connections between all surfaces (i.e. external faces bounding the original solid model and internal one inserted into this model). The main idea of our approach consists, at first, in the repairing of topological errors and in the rebuilding of disjoint regular sub-solids corresponding to 3D partitions of the original model. Once these independent sub-solids are recreated, they are assembled using a non-conventional partitioning operation to obtain a partitioned 3D model equivalent to the original non-manifold model.

Four main steps form our fully automatic method (Fig. 4):

Step 1: Transfer into SALOME ${ }^{\circledR}$ CAD module of the 3D CAD model using a neutral format (Fig. 4a).

Step 2: Restoration of the connectivity of the model using the repairing tools available into SALOME ${ }^{\circledR}$ (stitching of the faces, Fig. 4b) and filling of the data structure used to recover the topology of the geometric elements.

Step 3: Construction of the closed shells using an iterative propagation algorithm working on manifold as well as non-manifold entities (Fig. 4c).

Step 4: Construction of the regular sub-solids, and subsequently the non-manifold 3D partitions, from rebuilt shells (Fig. 4d).

The algorithms relative to these steps are detailed in the Sect. 4.2. They have been implemented as scripts, developed in Python language, directly inside $\mathrm{SALOME}^{\circledR}$, industrial $\mathrm{CAD} / \mathrm{mesh} / \mathrm{FE}$ analysis platform [6]. The SAL$\mathrm{OME}^{\circledR}$ platform has been chosen because of its open-

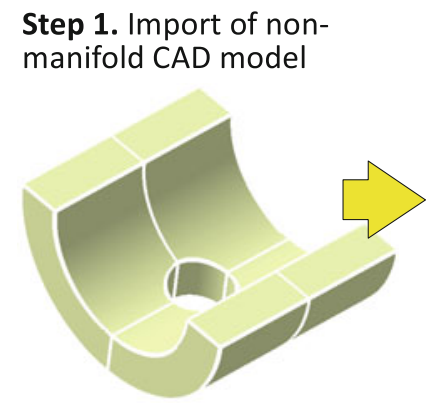

(a)
Step 2. Stitching of disconnected faces

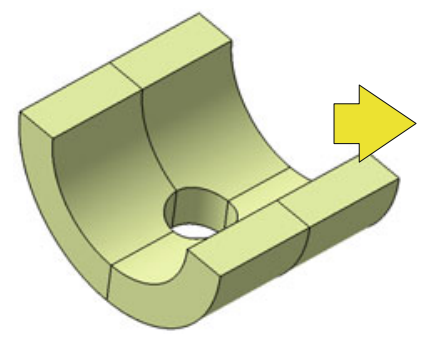

(b)
Step 3. Construction of closed shells

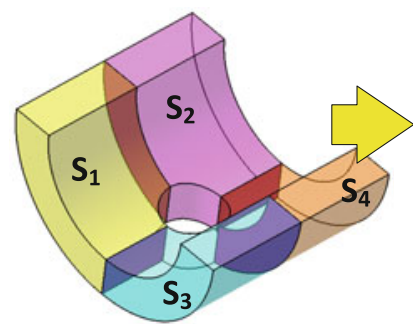

(c)
Step 4. Creation of 3D partitions

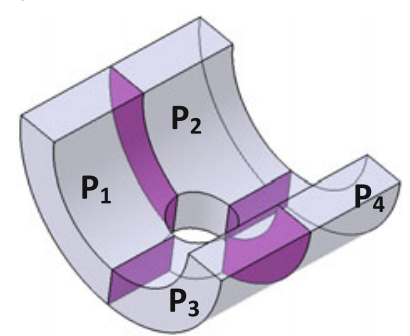

(d)

Fig. 4 Automatic reconstruction of 3D partitions based on an iterative propagation algorithm. a Import of non-manifold model as a set of disconnected faces. b Stitching disconnected faces. c Construction of closed shells from connected faces. d Creation of 3D partitions 


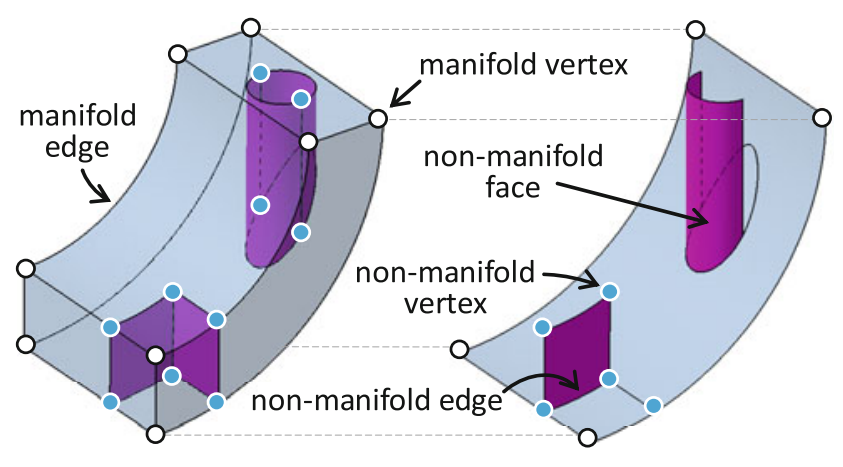

Fig. 5 Typology of the geometric entities

source character as well as the capability to manipulate non-manifold CAD models. The third step is the one that will be further detailed in the Sect. 4.2 since it gathers together the newly developed algorithm.

\subsection{Details of the proposed algorithm}

We suppose that after the transfer of partitioned 3D model (step 1 of our methodology), all information about connections between surfaces have been lost. Thus, during step 2 of the algorithm (see Sect. 4.1), the connections between faces are restored while looking for edges that can be merged. Two edges bounding two distinct faces can be merged if the confusion distance between them is smaller than a given threshold corresponding to a geometric modelling tolerance. Using this Euclidian distance criterion, nothing prevents the connection of more than two faces to a single edge. Thus, a specific data structure has been designed to store non-manifold configurations.

Once the data structure is filled in, edges and vertices can be tagged while following the sequencing below (Fig. 5):

(1) all the edges linking more than two faces, i.e. at least three faces, are tagged as non-manifold edges. All the other edges are considered as manifold edges.

(2) all the vertices that are connected to purely manifold edges are tagged as manifold vertices, whereas when at least one non-manifold edge is connected to a vertex, the vertex is tagged as a non-manifold vertex.

At this step of the process, we do not identify manifold faces from non-manifold faces since the notion of volume and inside/outside is not yet recovered.

During step 3, i.e. the key step of our approach (see Sect. 4.1), the automatic iterative reconstruction of shells propagates from the previously tagged manifold vertices according to the algorithm detailed in Fig. 6. The "secondary loop" of the algorithm allows creating closed shells from the set of connected faces. The principle is illustrated on the academic example of Fig. 7.
The search process of candidates to form future closed shells starts from a manifold vertex located on the "skin" of the object and considered as starting point to try to identify a new closed shell. The algorithm retrieves a set of faces connected to the starting point and expands them using the information about surface connections restored during step 2. The search of shells from object's skin avoids falling into problematic configurations such as nonuniqueness of solutions that is a typical problematic configuration occurring if the shell reconstruction begins from an internal surface contained in the imported geometry. The searching of new potentially closed shells propagates within the model by removing already discovered shells related to the object's skin, and this until all the faces of the imported model are ran. The propagation character of the shell reconstruction algorithm is clearly shown in Figs. 8 and 9: the searching of candidates to form closed shells corresponding to future partitions propagates from exterior (object's skin) to interior of the object. Thus, the proposed algorithm is of type heuristic in the sense that nothing ensure that the algorithm always succeed to close shells to form partitions. This is discussed in the Sect. 5.

The final step 4 of the algorithm creates a set of disconnected manifold sub-solids from a list of closed shells identified during step 3 . These sub-solids correspond to future 3D partitions present in the original non-manifold CAD model before its transfer. The last operation consists in the sub-solid association: all the created sub-solids are assembled into a non-manifold 3D model by maintaining the interfaces between sub-solids forming 3D partitions. The interfaces between reconstructed partitions correspond to internal faces common to two jointed sub-solids.

\section{Results and discussion}

The first example comes from an academic CAD model made of 27 cubic partitions, wherein one of the partitions is completely inside the model, i.e. not connected directly to the outer skin of the model. This model has been imported into SALOME ${ }^{\circledR}$ CAD module using the IGES protocol. The imported model contains, after its cleaning, 108 faces and 0 solids. The algorithm starts with the eight initial manifold vertices and closes directly eight cubic sub-solids after one iteration (Fig. 8a). In the second loop, 32 manifold vertices are identified and give rise to the definition of 12 sub-solids (Fig. 8b). In the third loop, 24 manifold vertices are identified and give rise to 6 sub-solids (Fig. 8c). Finally, a single sub-solid is identified starting from four non-manifold vertices (Fig. 8d). The last operation consisting of the sub-solid association allows creating 27 cubic partitions corresponding to 27 reconstructed sub- 
Fig. 6 Algorithm for automatic reconstruction of closed shells using an iterative propagation (corresponds to the step 3 of the proposed methodology)
Add face to CheckedFaces if all edges of face are connected to another face of CurrentFaces

End for

If (all edges of all CheckedFaces are connected to another face of CheckedFaces)

ShellClosure $=1$

End if

If (ShellClosure equal 0)

Add to CurrentFaces the faces not yet inside CurrentFaces but which are connected through edges to faces already in the CurrentFaces list

Else if (ShellClosure equal 1)

Create a shell with the faces of CurrentFaces

Create a sub-solid from the shell

Delete from the ManifoldVertices list all the vertices which are present into the previously created sub-solid

End if

End while

End while

Update the data structure by deleting the "skin" faces (faces which belong to only one shell) already placed into a shell and we update then the list ManifoldVertices until having checked the entire model solids. The overall algorithm takes $4 \mathrm{~s}$ on a $\mathrm{PC}$ with a $7 \mathrm{~GB}$ of RAM and a processor cadenced at $2.4 \mathrm{GHz}$.

Similarly, on the partitioned CAD model of a tube-like structure (courtesy EDF R\&D, Fig. 9) transferred using the IGES protocol, the algorithm finds 28 sub-solids, corresponding to 3D partitions, in $<10 \mathrm{~s}$ (same $\mathrm{PC}$ at $2.4 \mathrm{GHz}$ ). The exported non-manifold tube-like model contains 155 disjoint surfaces before stitching operation. However, here, eight central partitions have not been found by the algorithm, and a post-processing is still required in the current version of the algorithm. This algorithm is of type heuristic and nothing ensures that all the shells are recovered. This is due to the fact that the current algorithm solely uses the topology of the model to close the shell. As discussed in the conclusion, we plan to couple an octree-based data structure to better handle the notion of volume. Anyhow, if the partitions had to be created by hand starting from scratch, it would have taken about 1 day for an expert of SALOME $^{\circledR}$.

It can be noticed that the proposed algorithm assumes that there exists at least a starting vertex, i.e. a manifold vertex. The treatment of configurations wherein there would be no manifold vertex form future work.

The analysis of the quality of the resulting models allows validating our approach. The number and the shape of the automatically rebuilt 3D partitions are the key parameters allowing verifying the correct functioning of the algorithm. In the case of academic example (cube-like 


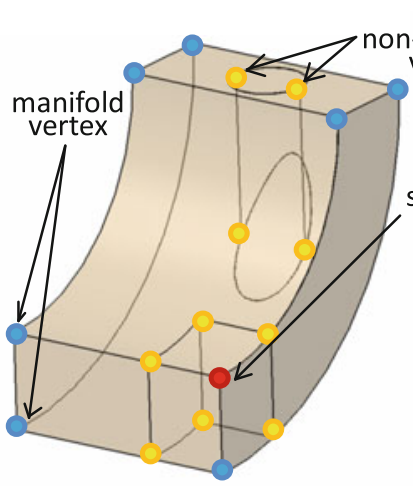

(a)

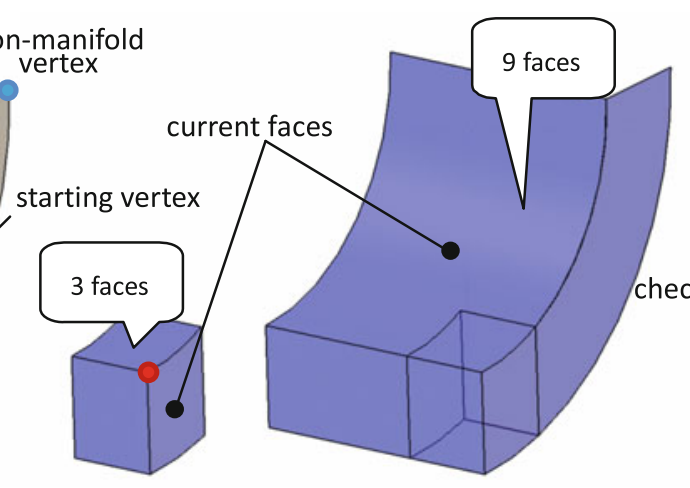

(b) (c)

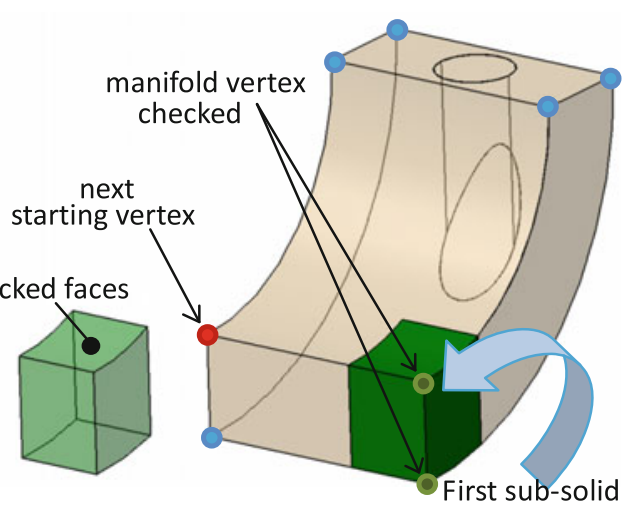

(d)

(e)

Fig. 7 Steps of the automatic 3D partition recovering on an academic example ("secondary loop" of the algorithm depicted on Fig. 6). a Choice of a starting point to begin the search process. b Searching of faces connected to the starting point. $\mathbf{c}$ Identification of joined faces

candidates to form the first closed shell. $\mathbf{d}$ Creation of the closed shell from checked faces. e Construction of the first regular sub-solid corresponding to 3D partition, and the choice of next starting point to restart the closed shell search process

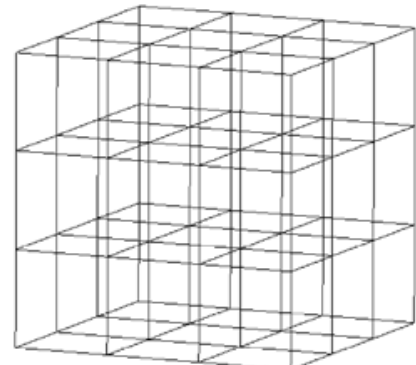

Initial model
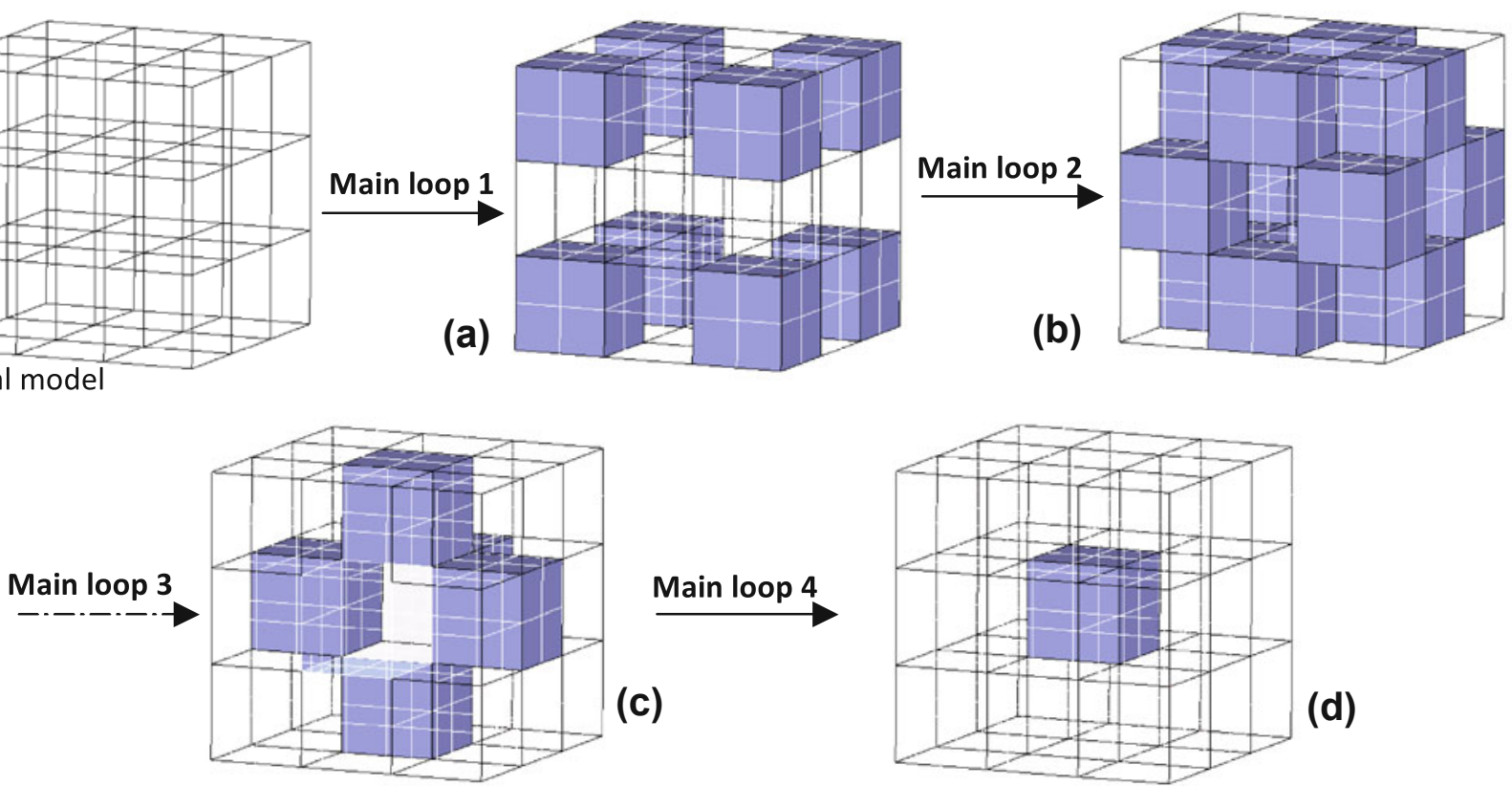

(c)

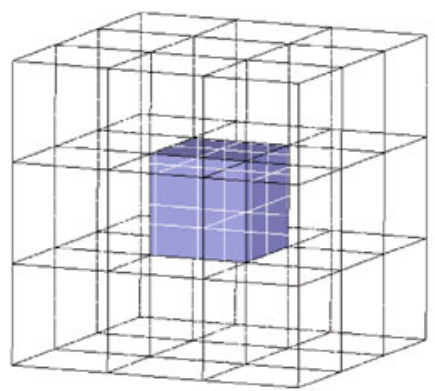

(d)

Fig. 8 Complete restoration of 27 cube-like 3D partitions from 108 disjoint faces by propagating the shell search and sub-solid construction process from exterior to interior of the geometric object

model), the number of reconstructed and original (i.e. before model transferring) partitions is identical. The shape of the rebuilt partitions is similar compared to original model. In addition, the measurement of volumes of the reconstructed and original models gives the same result.

In the case of industrial tube-like model, the number of obtained partitions represents $80 \%$ of the partitions containing in the original model (taking into account the discussed above limitations of the algorithm). The shape and volume of the recreated partitions are similar in comparison with the original partitions.
Figure 10 presents an example of industrial non-manifold CAD model that has been completely reconstructed by our algorithm. This is a partitioned 3D model of a "U-like" testing bench prototyped to qualify a measurement procedure required to validate a new design solution improving the mechanical behaviour of a valve used in power plants (courtesy EDF R\&D). The original partitioned model has been transferred using the IGES protocol. Starting from 222 disjoint surfaces, the algorithm finds 26 sub-solids and reconstructs the 26 joined three-dimensional partitions, initially presented in the original model, in $<29$ s. To be 


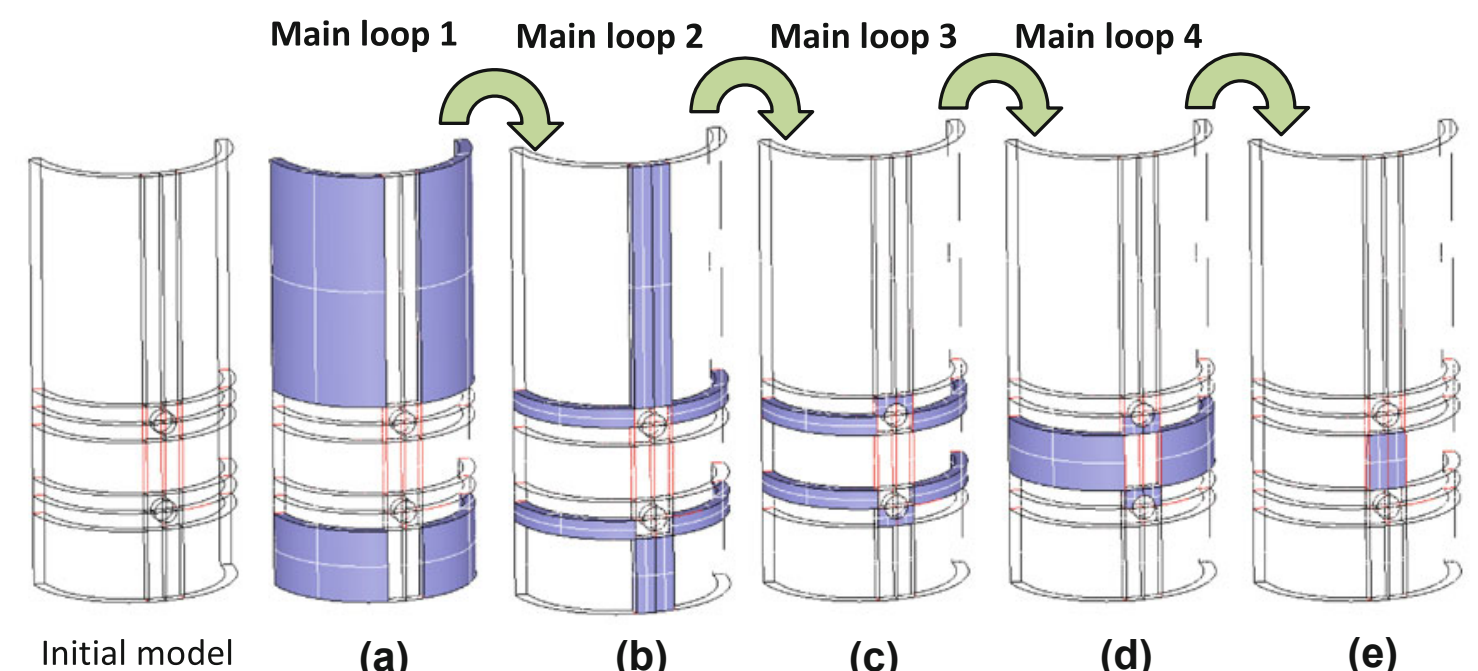

Fig. 9 Semi-complete restoration of 36 3D partitions from 155 disjoint surfaces on the CAD model of a tube-like structure (courtesy EDF R\&D)
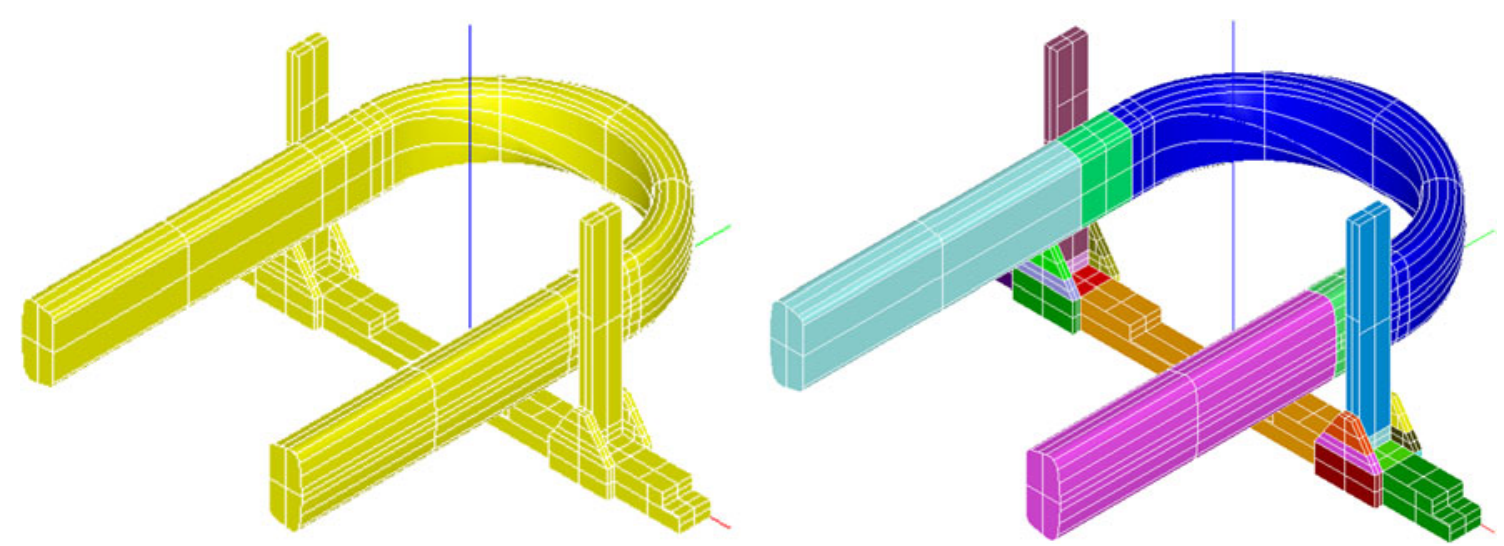

Fig. 10 Example of complete restoration of 26 3D partitions from 222 disjoint surfaces on the CAD model of a "U-like" testing bench (courtesy EDF R\&D)

Fig. 11 Example of a complete restoration of 56 partitions from 292 disjoint surfaces on the CAD model of a "tube-plate interaction" zoom model (courtesy EDF R\&D)
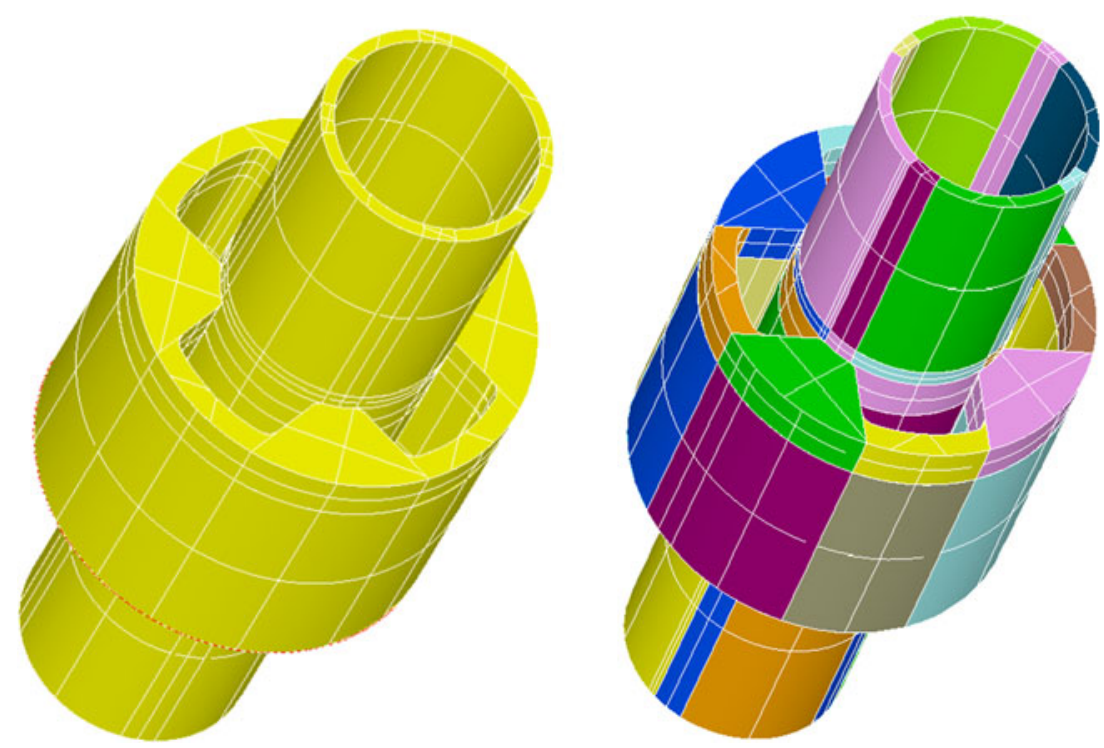
distinguished, the restored partitions are coloured in Fig. 10.

In addition, Fig. 11 illustrates another example of industrial non-manifold 3D CAD model- "tube-plate interaction" zoom model (courtesy EDF R\&D)—processed using the algorithm proposed in the paper. The original model contains 292 surfaces and 56 three-dimensional partitions required for:

- separation of the tube and the plate having different material properties,

- generation of the structured hexahedral mesh combined witch a local refinement,

- definition of the tube-plate interaction zones to simulate a non-linear multi-contact problem (the objective is to evaluate the lifecycle of the power production equipment).

The partitioned tube-plate interaction model has been transferred using the IGES protocol and completely reconstructed by our algorithm. Starting from 292 disjoint surfaces, the algorithm calculates all 56 sub-solids and reconstructs the 56 joined $3 \mathrm{D}$ partitions in $<52 \mathrm{~s}$. To be distinguished, the computed partitions are coloured in Fig. 11.

Finally, one can notice that, theoretically, there exists a limitation when in the secondary loop of the algorithm several faces can close simultaneously a shell and generate several sub-solids. In the proposed approach, shells are closed one by one and such a configuration is not handled. However, such theoretical configurations have not been encountered in all the industrial configurations that have been treated which restrict the impact of this restriction. The coupling to an octree-based approach should overcome this limit.

\section{Conclusion}

In this paper, we discuss typical problems encountered today when transferring non-manifold 3D models between different CAD systems. Starting from configurations identified as problematic from the industrial point of view, a methodology for reconstructing 3D partitions is proposed and successfully validated on academic and industrial models. The algorithm described in the paper allows restoring automatically partitions from a non-manifold $3 \mathrm{D}$ CAD model imported as a set of potentially disconnected entities (vertex, edges, and faces). The algorithm is iterative: it starts from the so-called manifold vertices and propagates along the repaired faces until shells close. We use a topological criterion of connectivity between geometric entities to identify the candidates for creation of shells. The shells are closed "as fast as possible" in the sense that the algorithm tends to minimise the number of faces used to close the shells. The set of identified closed shells represents the basis for the creation of the corresponding regular sub-solids and non-manifold 3D partitions. The proposed iterative reconstruction algorithm is of type heuristic. It saves a lot of time during the engineering design process. Partitions are not anymore reconstructed by hand starting from scratch but automatically. However, this is not the most efficient criterion. It may induce the creation of inaccurate shells.

To overcome these limits, we intend to couple an octree data structure to the existing topologic data structure, so that not only the topology but also the positioning of the geometric elements in 3D can be captured and used during the creation of new partitions. The algorithm also has to be adapted to handle configurations wherein there exists only non-manifold vertices at the beginning of the iterations.

Acknowledgments This work has been carried out under a research contract between the Research and Development Direction of the EDF Group and the Arts et Métiers ParisTech Aix-en-Provence.

\section{References}

1. Song H, Eynard B, Roucoules L, Lafon P, Charles S (2007) Beyond geometric CAD system: implementation of STEP translator for multiple-view product modeller. Int J Prod Lifecycle Manag 2(1):1-17

2. Gerbino S (2003) Tools for the interoperability among CAD systems. In: Proceedings of ADM-INGEGRAF International Conference on Tools and Methods Evolution in Engineering Design, Cassino, Naples and Salerno, Italy

3. Braid IC (1979) Notes on a geometric modeler, CAD Group Document no. 101, University of Cambridge

4. Lou R, Pernot JP, Mikchevitch A, Véron P (2010) Merging enriched finite element triangle meshes for fast prototyping of alternate solutions in the context of industrial maintenance. Comput Aided Des 42(8):670-681

5. Mikchevitch A, Geniaut S, Nistor I (2009) Towards fast numerical studies for maintenance and lifecycle problem analysis: new simulation methods on example of an industrial study case. In: Proceedings of ASME international pressure vessels and piping conference, design and analysis, vol 3, Prague, Czech Republic, pp 253-262. doi:10.1115/PVP2009-77154

6. Salome ${ }^{\circledR}$ Open Source Integration Platform for Numerical Simulation (2012). http://www.salome-platform.org

7. Adzhiev V, Kartasheva E, Kunii T, Pasko A, Schmitt B (2002) Cellular-functional modeling of heterogeneous objects. In: ACM symposium on solid modeling and applications, Saarbrucken, Germany, pp 192-203

8. Kumar V, Dutta D (1997) An approach to modeling multimaterial objects. In: ACM symposium on solid modeling and applications, Atlanta, USA, pp 336-345

9. Rossignac JR (1994) Through the cracks of the solid modeling milestone. In: Coquillart S, Strasser W, Stucki P (eds) From object modelling to advanced visualization. Springer, Berlin, pp 1-75

10. Rossignac JR, Requicha AAG (1991) Constructive non-regularized geometry. Comput Aided Des 23(1):21-32 
11. Chen JM, Gursoz EL, Prinz FB (1993) Integration of parametric geometry and non-manifold topology in geometric modeling. In: ACM symposium on solid modeling and applications, Montreal, Canada, pp 53-64

12. Dobkins DP, Laszlo MJ (1987) Primitives for the manipulation of three-dimensional subdivisions. In: ACM symposium on computational geometry, Waterloo, Canada, pp 86-99

13. Vanecek G, Nau D (1987) Non-regular decomposition: an efficient approach for solving the polygon intersection problem. In: Proceedings of symposium on integrated and intelligent manufacturing, ASME Winter Annual Meeting, pp 271-279

14. Weiler K (1988) The radial edge structure: a topological representation for non-manifold geometric boundary modeling. In: Encarnacao JL, Wozny MJ, McLaughlin HW (eds) Geometric modelling for CAD applications. North-Holland, Elsevier Science, Amsterdam, pp 3-36

15. De Floriani L, Hui A (2003) A scalable data structure for threedimensional non-manifold objects. In: Eurographics symposium on geometry processing, Aachen, Germany, pp 73-83

16. Desaulnier H, Stewart N (1992) An extension of manifold boundary representation to R-sets. ACM Trans Graph 11(1): $40-60$

17. Rossignac JR, O'Connor M (1989) SGC: a dimension-independent model for point sets with internal structures and incomplete boundaries. In: Wosny M, Turner J, Preiss K (eds) Proceedings of the IFIP Workshop on CAD/CAM, geometric modeling for product engineering. North-Holland, Amsterdam, pp 145-180

18. Hui A, Vaczlavik L, De Floriani L (2006) A decomposition-based representation for 3D simplicial complexes. In: Eurographics symposium on geometry processing, Aire-la-Ville, Switzerland, pp 101-110

19. Marcheix D, Gueorguieva S (1998) Nibble meshing: incremental triangulation of non-manifold solid boundary. Comput Graph 22(2-3):181-188

20. Saxena M, Finnigan PM, Graichen CM, Hathaway AF, Parthasarathy VN (1995) Octree-based automatic mesh generation for non-manifold domains. Eng Comput 11(1):1-14

21. Zhang Y, Hughes TJR, Bajaj CL (2010) An automatic 3D mesh generation method for domains with multiple materials. Comput Methods Appl Mech Eng 199(5-8):405-415

22. Qian J, Zhang Y (2012) Automatic unstructured all-hexahedral mesh generation from B-Reps for non-manifold CAD assemblies. Eng Comput 28(4):345-359

23. Gu H, Chase TR, Cheney DC, Bailey T, Johnson D (2001) Identifying, correcting and avoiding errors in CAD models which affect interoperability. ASME J Comput Inf Sci Eng 1(2): $156-166$

24. Krause FL, Stiel C, Liiddemann J (1997) Processing of CADdata-conversion, verification and repair. In: ACM symposium on solid modelling and applications, Atlanta, USA, pp 248-254

25. Ficco MM, Mandorli F, Otto HE (1999) Error classification and recovery within CAD models reconstruction. In: ACM symposium on solid modelling and applications, Ann Arbor, USA, pp 316-317

26. Gerbino S, Crocetta S, Di Martino C (1997) Data exchange in CAD systems: limits, solutions, perspectives. In: Proceedings of ADM international conference on design tools and methods in industrial engineering, Florence, Italy, pp 423-434

27. Sangole A, Knopf GK, Mak H (1999) Micro geometry flaws in the exchange of design data using STEP. Int J Concurr Eng Res Appl 7(4):335-345

28. Mortenson MM (1985) Geometric modeling. Webster J \& Sons, New York

29. Mezentsev A, Woehler T (1999) Methods and algorithms of automated CAD repair for incremental surface meshing. In: Proceedings of the 8th international meshing roundtable, South Lake Tahoe, USA, pp 299-309

30. Ju T (2009) Fixing geometric errors on polygonal models: a survey. J Comput Sci Technol 24(1):19-29

31. Barequet G, Kumar S (1997) Repairing CAD models. In: IEEE Conference Visualization'97, pp 363-370

32. Barequet G, Duncan CA, Kumar S (1998) RSVP: a geometric toolkit for controlled repair of solid models. IEEE Trans Vis Comput Graph 4(2):162-177

33. Bohn JH, Wozny MJ (1993) A topology-based approach for shell-closure. In: Wilson et al (eds) Conference on geometric modeling for product realization, Amsterdam, The Netherlands, pp 297-319

34. Veleba D, Felkel P (2007) Survey of errors in surface representation and their detection and correction. In: Proceedings of the 15 th international conference in central Europe on computer graphics (WSCG'07), Plzen-Bory, Czech Republic, pp 65-72

35. Yang J, Han S (2006) Repairing CAD model errors based on the design history. Comput Aided Des 38:627-640

36. Bargier A, Mikchevitch A, Pernot JP (2011) Automatic reconstruction of 3D partitions from exported non-manifold models. In: Fischer X, Nadeau JP (eds) Proceedings of international conference IDMME-virtual concept 2010, research in interactive design, vol 3. Springer, Berlin, pp 63-64 